\title{
La autonomía informativa en Comunicación Nuclear: análisis de la legislación vigente
}

\section{Informational autonomy in Nuclear Communication: analysis of current legislation}

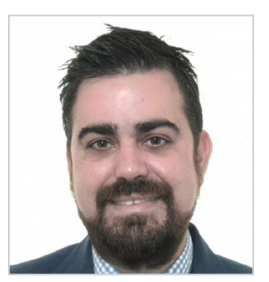

Enrique Cobos Urbina. Licenciado en Comunicación Audiovisual por la Universidad de Navarra (2004), Máster en Radio por la Universidad CEU San Pablo (2006), Máster en Dirección de Comunicación Empresarial e Institucional por la Universidad Autónoma de Barcelona (2012) y doctor en Periodismo por esta universidad (2018). Autor de diversas publicaciones sobre comunicación, su investigación se ha centrado en la comunicación estratégica en sectores en sectores de riesgo, especialmente en el sector atómico. Ha trabajado en varios medios de comunicación en España y en la actualidad es responsable de comunicación del Museo de Ciencias y de la Facultad de Ciencias de la Universidad de Navarra y profesor de Comunicación Ambiental y Expresión Escrita en esta institución.

Instituto de Biodiversidad y Medioambiente. Universidad de Navarra, España

ecobos@unav.es

ORCID: 0000-0002-6556-0127

Recibido: 30/04/2020 - Aceptado: 16/11/2020

\section{Resumen:}

La opinión pública en España se ha posicionado históricamente en contra de la energía nuclear. El sector atómico señala la falta de información y el desconocimiento de la población como las causas principales de ese rechazo. El puente que une a la opinión pública y a la industria atómica debe trazarse con información transparente por parte de la autoridad responsable de informar (ya sea el Gobierno, el organismo regulador, o una central nuclear) para que los ciudadanos estén protegidos en caso de sucesos imprevistos, y para que puedan comprender las ventajas e inconvenientes de esta fuente de energía. En este sentido, la legislación en materia de Comunicación Nuclear contempla la transparencia como la base fundamental para esa interacción con la sociedad. En este artículo se revisa la normativa internacional, europea y nacional para conocer la proyección e influencia que la regulación tiene en la gestión de la comunicación de estas industrias.

\section{Palabras clave:}

Transparencia; legislación nuclear; Comunicación Nuclear; energía nuclear; central nuclear.

\section{Received: 30/04/2020 - Accepted: 16/11/2020}

\section{Abstract:}

Public opinion in Spain has historically been opposed to nuclear energy. The atomic sector points to a lack of information and awareness among the population as the main causes of this rejection. Bridges between public opinion and the atomic industry must be built with transparent information by those authorities with a responsibility to inform (be they the government, the regulatory body, or a nuclear power plant) so that citizens are protected in case of unforeseen events, and to ensure they understand the advantages and disadvantages of this energy source. In this sense, the legislation on Nuclear Communication considers transparency to be the fundamental basis for this interaction with society. In this article we review the international, European and national regulations to have a clear idea of the projection and influence that this regulation has on the communication management of these industries.

\section{Keywords:}

Transparency; nuclear legislation; Nuclear Communication; nuclear energy; nuclear power plant.

\footnotetext{
Cómo citar este artículo:

Cobos Urbina, E. (2020). La autonomía informativa en Comunicación Nuclear: análisis de la legislación vigente. Doxa Comunicación, 31, pp. 153-166.
}

https://doi.org/10.31921/doxacom.n31a7 


\section{Introducción}

Los resultados de las encuestas de opinión son contundentes respecto a la aceptación pública de la energía nuclear y sus centrales de producción: en los últimos años los españoles están en contra de la energía nuclear aunque aumenta su aceptación cuando tienen un mayor conocimiento sobre algunos aspectos de esta fuente de energía (Ipsos Consulting e Innova Investigación de Mercados, 2014-2018).

Los españoles, desde la implantación de la energía nuclear en los años 60, han percibido maniobras de opacidad y falta de transparencia por parte de los gobiernos y de las empresas que operan las centrales nucleares. Sumado a esto, el desconocimiento de la población, la mala gestión de algunos incidentes en España, las deficiencias comunicativas en los accidentes civiles (Three Mile Island, Chernóbil y Fukushima), las campañas antinucleares, el posicionamiento ideológico de líderes políticos y de opinión, la incertidumbre en la gestión de los residuos radioactivos, y el miedo a un posible accidente nuclear son causas principales de esa desafección de la población ante la energía nuclear, como reflejan los principales autores en esta materia y algunos sondeos del CIS y eurobarómetros ${ }^{1}$.

Estas encuestas reflejan además que la seguridad, el medio ambiente, la salud pública, los accidentes, la rentabilidad en la producción y los residuos radioactivos son los temas que han coronado el debate históricamente y de una manera cíclica. En la actualidad el debate en España gira en torno al cierre de las plantas atómicas de este país o la renovación de sus licencias de explotación ${ }^{2}$.

¿Son los Gobiernos responsables cuando informan sobre la conveniencia en la utilización de esta fuente de energía? ¿El organismo regulador traslada una información transparente a la población sobre los sucesos en las plantas atómicas? ¿Las centrales nucleares supeditan sus propios intereses en favor de un conocimiento real del sector? ¿Están los ciudadanos informados respecto a la energía nuclear? ¿Qué obligaciones informativas tienen las autoridades en el sector atómico? En España, el derecho a la información está contemplado en el artículo 20 de la Constitución de 1978.

El objetivo de este estudio es conocer qué autonomía real tienen las centrales nucleares para comunicarse con el público y qué restricciones legales tienen estas industrias en materia de comunicación. Además, una vez conocida la normativa en Comunicación Nuclear, pretendemos saber si los ciudadanos están protegidos por la ley a nivel informativo y en qué situaciones.

\section{Metodología}

Para esta investigación planteamos las siguientes cuestiones:

¿Las centrales nucleares de España tienen restricciones legales en materia de comunicación o poseen autonomía para comunicarse con sus públicos en cualquier situación?

1 Cfr. Sondeos CIS (febrero 1995, noviembre 1997, diciembre 2000, noviembre 2007, mayo 2011) y Eurobarómetros (diciembre 2002, septiembre 2005, enero 2006, febrero 2007).

2 En 2020 las centrales nucleares de Vandellós II y Almaraz han renovado su licencia de explotación por un período de diez y ocho años respectivamente. 
¿Los ciudadanos están amparados por la ley a nivel informativo en asuntos nucleares?

Para poder responder a estas preguntas hemos desarrollado la siguiente metodología. Por una parte hemos revisado la legislación nuclear existente (internacional, europea y nacional) para conocer cuáles son las limitaciones normativas que el derecho nuclear impone a las centrales nucleares en materia de comunicación. De esta manera pretendemos conocer qué autonomía real tienen las instalaciones nucleares españolas para comunicarse con sus públicos en las diferentes situaciones, es decir, tanto en períodos de actividad ordinaria como en momentos de crisis. Además, pretendemos conocer si los ciudadanos están protegidos por la ley a nivel informativo y en qué situaciones.

Por otra parte, hemos entrevistado en profundidad a los responsables de comunicación de las plantas atómicas de España para conocer de primera mano cuál es su visión sobre la transparencia nuclear y cómo interpretan ellos la función comunicativa y las limitaciones normativas ${ }^{3}$.

Las entrevistas se han realizado de manera presencial en cada una de las sedes oficiales: Madrid, Tarragona y Valencia. Los profesionales entrevistados han sido Monserrat Godall (Asociación Nuclear Ascó-Vandellós II. 3 de noviembre de 2016), Antonio Cornadó (C.N. de Santa Ma de Garoña y Foro de la Industria Nuclear Española. 21 de diciembre de 2016), Juan Pedro Alcázar (Centrales Nucleares Almaraz-Trillo. 21 de diciembre de 2016), Jesús Cruz (C.N. de Cofrentes. 16 de febrero de 2017), y Javier Sala y Carlos Gómez (C.N. de Cofrentes. 17 de febrero de 2017). Además, se realizó una entrevista por correo electrónico a Antonio Melo (Centrales Nucleares Almaraz-Trillo. 25 de enero de 2017).

Las entrevistas presenciales no han superado las dos horas de duración y han sido grabadas y transcritas. Se ha empleado un mismo cuestionario dividido en seis bloques temáticos: la comunicación en la industria nuclear; el departamento de comunicación de la central y su política de comunicación; la comunicación de la central en situaciones regulares; la comunicación de crisis en la industria nuclear; el responsable de comunicación de la central; y la central nuclear y la opinión pública.

Además, hemos examinado la literatura existente sobre Comunicación Nuclear. Aunque no existe un desarrollo doctrinal sobre cómo tienen que ejercer la comunicación las centrales nucleares en situaciones de actividad ordinaria -más allá de algunas recomendaciones de organismos oficiales del sector como el Organismo Internacional de la Energía Atómica o el Consejo de Seguridad Nuclear- algunos autores han relacionado determinados aspectos de la comunicación con situaciones de crisis nuclear. Las principales aportaciones son de Cobos \& Recoder (2019a), De Oliveira (2015), Koerner (2014), Siegrist y Visschers (2013), Abe (2013), Lu (2012), Yamamura (2012) Ionescu (2012), Perko (2011) o Perko et al. (2012, 2013). Por último, para esta investigación se han revisado todos los eurobarómetros (especiales, flash y estándar) desde 1974 hasta 2019 para conocer la evolución de la opinión de los europeos sobre la actividad nuclear y los temas más importantes que influyen en su percepción. Los estudios de opinión pública en Europa que atañen al sector atómico son: octubre de 2001 , marzo de 2010, junio de 2008, febrero de 2007, enero de 2006, septiembre de 2005, diciembre y abril de 2002. Por otra parte, hemos revisado los sondeos del Centro de Investigaciones Sociológicas (CIS) desde 1979 hasta 2019 para conocer

3 Para esta investigación hemos tenido en cuenta a la central nuclear de Santa María de Garoña (Burgos) ya que, a pesar de que cerró definitivamente en 2017, el testimonio de Antonio Cornadó -que estuvo más de 20 años como director de comunicación de la central y posteriormente como presidente del Foro Nuclear- es relevante y enriquecedor para nuestro objeto de estudio. 
con qué regularidad se formulan preguntas respecto a la energía nuclear y qué percepción tienen los españoles respecto a la producción de electricidad de este origen. También hemos analizado los estudios de opinión pública y energía nuclear en España que cada año realiza Ipsos Consulting e Innova Investigación de Mercados, a petición del Foro de la Industria Nuclear Española (2004-2018), y a los que hemos tenido acceso.

\section{Resultados}

\subsection{Restricciones normativas en Comunicación Nuclear}

La legislación nuclear es amplia y no contempla una normativa desarrollada e impositiva sobre cómo tienen que comunicarse las centrales nucleares con sus grupos de interés en situaciones regulares.

\subsubsection{Marco internacional}

A nivel internacional existen tratados o declaraciones -algunos no vinculantes- que suscriben los Estados. El Convenio de Aarhus fue firmado el 25 de junio de 1998 en Dinamarca por la Unión Europea y 51 Estados de Europa y Asia. Este tratado internacional garantiza el derecho de los ciudadanos a la información, participación en la toma de decisiones y el acceso a la justicia en materia de medio ambiente.

Aarhus es un tratado internacional que afecta al sector nuclear. En su artículo 4 se recoge el derecho de los ciudadanos a estar informados en temas de medio ambiente ${ }^{4}$ por las autoridades públicas ${ }^{5}$, y la responsabilidad que éstas tienen de informar, de manera transparente, sobre el medio natural ${ }^{6}$.

En el artículo 5 de Aarhus se indica también la obligación de una difusión inmediata de información a aquellas personas que puedan resultar afectadas por cualquier amenaza para la salud o el medio ambiente, motivado por causas naturales o actividades humanas.

Aarhus es una herencia de la Declaración de Río ${ }^{7}$ de 1992. En el artículo 10 de la carta brasileña se apela a la transparencia informativa de los Estados en materia medioambiental:

"En el plano nacional, toda persona deberá tener acceso adecuado a la información sobre el medio ambiente de que dispongan las autoridades públicas, incluida la información sobre los materiales y las actividades que encierran peligro en sus comunidades, así como la oportunidad de participar en los procesos de adopción de decisiones. Los Estados deberán

4 En el artículo 2 se especifica qué se entiende sobre información de medio ambiente. La energía, las radiaciones y la salud de las personas entran dentro de esta categoría.

5 En el artículo 2 se define "Autoridad Pública" como administración pública nacional, regional y local pero, además, como todas aquellas personas físicas o jurídicas que presten servicios públicos o tengas funciones públicas relacionadas con el medio ambiente. Se entiende que las centrales nucleares entrarían dentro de esta definición.

6 Aarhus solo permite negar el acceso a la información ambiental en determinados casos que puedan afectar a la confidencialidad, a la seguridad pública, y situaciones determinadas que estén sujetas a un procedimiento judicial.

7 La Declaración de Río de Janeiro fue una cumbre mundial sobre Medio Ambiente y Desarrollo organizada por las Naciones Unidas en Brasil del 3 al 14 de junio de 1992. 
facilitar y fomentar la sensibilización y la participación de la población poniendo la información a disposición de todos. Deberá proporcionarse acceso efectivo a los procedimientos judiciales y administrativos, entre éstos el resarcimiento de daños y los recursos pertinentes".

El artículo 18 de esta declaración exige a los Estados que informen de manera inmediata en caso de desastres y situaciones de emergencia que pudieran afectar al medio ambiente.

Veinte años después de aquella reunión, se celebró en Río de Janeiro, la Conferencia de Naciones Unidas sobre Desarrollo Sostenible que reafirmó los temas de Río‘92 en lo que concierne a nuestro objeto de estudio.

La Declaración de Río es considerada soft law ya que la responsabilidad de los Estados no va más allá de un compromiso moral o de una declaración de intenciones. Sin embargo, Aarhus tiene el carácter obligatorio para los países que lo suscriben $^{10}$.

\subsubsection{Marco europeo}

En Europa, la implantación de las directivas es normativa para los Estados miembros de la Unión Europea. Es competencia exclusiva de los Estados la seguridad de las centrales nucleares y la gestión del combustible gastado.

Con el nacimiento del Tratado Euratom ${ }^{11}$, la Unión Europea ha creado directivas sobre energía nuclear, con el objetivo de proteger a los ciudadanos europeos de la radiación y de salvaguardar el medio ambiente.

En la última directiva europea sobre energía nuclear y seguridad se establecen las normas básicas en seguridad nuclear para los Estados miembros de la Unión Europea. En esta directiva del año 2014 se recogen también algunas normas generales referentes a la comunicación con el público. En el artículo 8 se aboga por la transparencia informativa de los Estados -en materia de seguridad de las plantas- hacia los grupos de interés principales donde operan las centrales, esto es, poblaciones cercanas, autoridades locales y trabajadores de la planta nuclear.

Además, se establece como obligación la información a los trabajadores de la central, autoridades locales y público en general por parte de los titulares de las nucleares y organismos competentes en materia de regulación, no solo en la operación ordinaria de las centrales, sino también una información rápida a estos stakeholders en caso de accidentes o incidentes.

Esta transparencia informativa exigida a los Estados miembros puede estar vinculada a la aceptación de la energía nuclear por parte de la opinión pública, como señala Ruiz de Apodaca (2010:9): “Transparencia, información y participación en la toma de estas decisiones es lo que hace generar la confianza necesaria entre el público, la industria nuclear y los reguladores para posibilitar el ejercicio de tales actividades".

8 Rio+20 se desarrolló en junio de 2012 en Río de Janeiro (Brasil). Los países mostraron de nuevo su compromiso en dos temas centrales: desarrollo sostenible y erradicación de la pobreza.

9 Disposiciones sin carácter normativo ni vinculante a los Estados que las suscriben.

10 Por ejemplo, España ratificó el Convenio en 2004 y entró en vigor en 2005. En el año 2006 nace la ley 27/2006 que asume Aarhus.

11 La Comunidad Europea de la Energía Atómica (EURATOM) nace como consecuencia del Tratado de Roma (1957). El Tratado Euratom establece las bases para el desarrollo de la energía nuclear en Europa. 
Cornadó (2006:34) también menciona la transparencia que la legislación otorga al sector nuclear: "La normativa en materia nuclear prevé desde sus inicios un completo y complejo sistema de información sobre todo el proceso industrial relacionado con la generación eléctrica nuclear. Este tipo de información otorga un grado de transparencia significativo a las actividades del sector".

Muñoz (2012:35), periodista del equipo de comunicación del Consejo de Seguridad Nuclear, también incide en la misma idea: "Se debe estar preparado para responder a una sociedad que reclama no sólo que las cosas se hagan bien sino que también se transmitan con transparencia. Y es que la transparencia y la comunicación son asuntos cruciales en la sociedad de la información que ha traído consigo el siglo XXI".

En este sentido, podemos decir que el punto de partida en la construcción de la confianza del público en materia nuclear, puede encontrarse en una información transparente por parte de las autoridades nucleares de cada Estado a los ciudadanos, en los temas que les preocupan en materia nuclear (seguridad, salud y medio ambiente).

Otra de las directivas europeas clave en materia nuclear es la que hace referencia a los residuos radioactivos y a la gestión del combustible gastado ${ }^{12}$ (2011). Una de las apuestas de esta directiva es suministrar información efectiva y establecer un diálogo con el público en favor de la transparencia.

\subsubsection{Marco español}

España, como país miembro de la Unión Europea, cumple con estas directivas en materia nuclear y debe asumir, en su totalidad, todo lo referente a la información al público y a la transparencia.

Si hacemos una revisión histórica de la legislación nuclear en España para ver el compromiso normativo que existe entre el Estado y los españoles -en lo que concierne a la información suministrada a la opinión pública-, podemos decir que, en la ley sobre energía nuclear de $19644^{13}$ (LEN a partir de ahora), no se hace referencia a la necesidad de información de las autoridades competentes en materia nuclear (Ministerio, Junta de la Energía, o centrales nucleares) hacia los ciudadanos españoles.

La LEN es el fundamento base sobre el desarrollo de la energía nuclear en España y, a pesar de que esta ley ha sufrido varias modificaciones ${ }^{14}$, no se contemplan indicaciones específicas a las centrales nucleares sobre cómo tienen que gestionar y organizar su comunicación con el público.

En las entrevistas realizadas para esta investigación se señala que las centrales nucleares tienen una comunicación reglada al Consejo de Seguridad Nuclear ${ }^{15}$ (CSN a partir de ahora), es decir, deben informar sobre los sucesos que tengan lugar

12 La Directiva 2011/70/Euratom obliga a cada Estado miembro a crear un almacén de residuos radioactivos en su territorio y a disponer de un organismo regulador independiente (En España es el Consejo de Seguridad Nuclear).

13 La Ley 25/1964 sobre Energía Nuclear es un compendio preconstitucional de 97 artículos en el que se pone en marcha el desarrollo de los usos pacíficos de la energía nuclear en España.

14 Ley 33/2007, Ley 11/2009, Ley 12/2011, Ley 15/2012.

15 El Consejo de Seguridad Nuclear es un organismo independiente del Estado con jurisprudencia propia y la autoridad competente en materia de regulación, vigilancia y control nuclear en España. 
en la instalación ${ }^{16}$ : "Nosotros cualquier cosa que se produzca -por mínima que sea- tenemos que notificarla" (Alcázar, 2016). Estos sucesos notificados pueden ser a una hora y a 24 horas. En el primer caso suelen ser sucesos más relevantes desde el punto de vista de la operación y van asociados a una nota de prensa por parte de las nucleares. En este caso, el CSN también comunica el incidente por sus medios a los grupos de interés.

A pesar de que las centrales nucleares comuniquen al organismo regulador cualquier incidente operativo en la planta y cuelguen en su página web estos sucesos notificados, la comunicación con el público está protocolizada en caso de accidentes (Cobos \& Recoder (2019a).

Por otra parte, el Consejo de Seguridad Nuclear -organismo independiente de la Administración General del Estado- sí que recoge entre sus funciones informar a la opinión pública a través de los medios de comunicación, publicaciones, Centro de Información, Internet o Twitter ${ }^{17}$. Este compromiso del CSN con la opinión pública se ve reflejado en la Ley de su creación (15/1980) y también en los objetivos de su Plan Estratégico 2017-2022 en los que se concibe la construcción de la credibilidad como la confianza que los ciudadanos españoles tienen en el Consejo para protegerles de la radiación, y sin influir en su sentimiento nuclear o antinuclear, como señala Muñoz (2012:34).

La transparencia es un objetivo del CSN y se traduce en facilitar a los ciudadanos el acceso a la información de tal manera que entiendan el proceso regulador, intentando reducir la asimetría en la información. El CSN entiende que, como organismo competente, dispone de una información más precisa que las autoridades nacionales y la opinión pública española.

La instrucción IS-19 del CSN del 22 de octubre de 2008 sobre los requisitos del sistema de gestión de las instalaciones nucleares tiene dos apuntes interesantes para este estudio. En el quinto punto -que hace referencia a las responsabilidades del personal directivo- se aborda la importancia de tener satisfechos a los grupos de interés (5.2): "La alta dirección tendrá en cuenta las expectativas de los grupos de interés en las actividades e interacciones en los procesos del sistema de gestión, con el fin de aumentar el grado de satisfacción de los grupos de interés y de garantizar al mismo tiempo que la seguridad no se vea comprometida".

Por otra parte, el punto siete de esta instrucción trata sobre la ejecución e implantación de diferentes procesos y aborda el tema comunicativo (7.3.16): "La información relevante para los objetivos de seguridad, de prevención de riesgos laborales, de protección medioambiental, de protección física, de calidad y económicos, se comunicará a las personas de la organización y, cuando sea necesario, a otros grupos de interés".

Otro compromiso de las centrales nucleares españolas a nivel normativo, es seguir las directrices del Plan Básico de Emergencia Nuclear ${ }^{18}$ (PLABEN) y la implantación, en caso de accidentes o incidentes, de sus planes específicos ${ }^{19}$ :

16 Las instrucciones del Consejo (IS-10) marcan aquellos aspectos que las centrales deben notificar al organismo regulador.

17 El CSN es el único organismo en España en materia nuclear -junto con el Foro Nuclear Español y la Sociedad Nuclear Española- que dispone de una cuenta en esta red social desde marzo de 2011: @CSN_es.

18 El PLABEN fue aprobado por Real Decreto el 25 de junio de 2004 y está vigente en la actualidad. Esta guía directiva contiene normas para actuar de acuerdo con los planes de emergencia nuclear de Protección Civil y es competencia de la Administración General del Estado.

19 El Consejo de Ministros acordó en al año 2006 los planes de emergencia exteriores y específicos de las centrales españolas, denominados según la distribución geográfica de las plantas: Burgos (PENBU), Cáceres (PENCA), Guadalajara (PENGUA), Tarragona (PENTA) y Valencia (PENVA). 
Tabla 1. Plan de Emergencia Nuclear Exterior por central

\begin{tabular}{|l|l|l|l|l|l|}
\hline PEN & Provincia & Central & $\begin{array}{l}\text { Acuerdo Consejo de } \\
\text { Ministros }\end{array}$ & $\begin{array}{l}\text { Fecha } \\
\text { aprobación }\end{array}$ & $\begin{array}{l}\text { Publicación } \\
\text { BOE }\end{array}$ \\
\hline PENBU & Burgos & Santa Ma de Garoña & $16 / 10 / 09$ & $20 / 10 / 09$ & $10 / 11 / 09$ \\
\hline PENTA & Tarragona & Ascó y Vandellós II & $16 / 10 / 09$ & $20 / 10 / 09$ & $10 / 11 / 09$ \\
\hline PENVA & Valencia & Cofrentes & $16 / 10 / 09$ & $20 / 10 / 09$ & $10 / 11 / 09$ \\
\hline PENCA & Cáceres & Almaraz & $16 / 10 / 09$ & $20 / 10 / 09$ & $10 / 11 / 09$ \\
\hline PENGUA & Guadalajara & Trillo & $16 / 10 / 09$ & $20 / 10 / 09$ & $10 / 11 / 09$ \\
\hline
\end{tabular}

Fuente: Consejo de Seguridad Nuclear: www.csn.es

Estos planes de respuesta exterior (PEN) recogen entre sus funciones -durante la emergencia- la información a la población afectada, a la Administración Pública y a los medios de comunicación. Cuando se produce la emergencia, el director del $\mathrm{PEN}^{20}$ dispondrá de un gabinete de comunicación encargado de informar a los grupos de interés sobre los riesgos que pudiera conllevar el accidente, las medidas de protección y sobre el propio plan de emergencia. Este gabinete estará integrado por personal de la delegación del Gobierno donde se ubique la planta nuclear y podrá incorporarse personal especializado en materia de información de las comunidades autónomas afectadas, así como expertos de la central nuclear $^{21}$ (Cobos \& Recoder (2019a).

\section{Gráfico 1. Mapa de la legislación en Comunicación Nuclear}

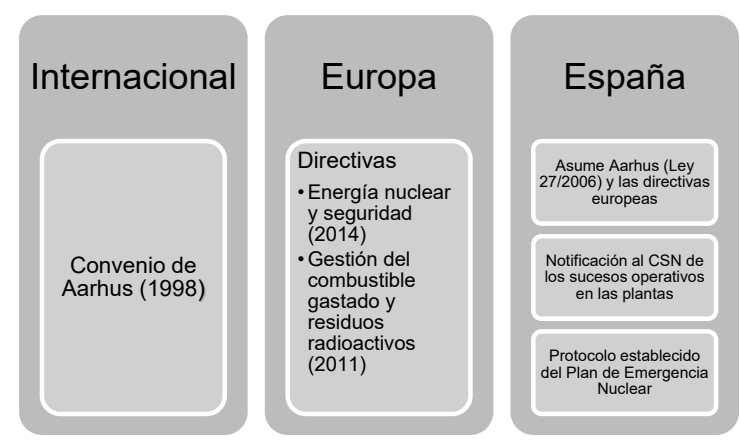

Fuente: elaboración propia

20 Según se especifica en el PLABEN (Título III), este cargo lo asumirá el Delegado del Gobierno de la Comunidad Autónoma donde esté ubicada la central nuclear.

21 En la ejecución del programa de información previa a la población participan además: los grupos operativos del PEN; los directores de los PAMEN; Ministerio de Sanidad y Consumo; el CSN y los órganos competentes de las Comunidades Autónomas afectadas y del Ministerio del Interior. 


\subsection{Praxis comunicativa de las centrales nucleares en España y recomendaciones de organismos oficiales.}

En España, la comunicación en situaciones de actividad ordinaria es competencia de las centrales nucleares, de las agrupaciones de interés económico o de las empresas propietarias que las respaldan -según el caso concreto-, siempre cumpliendo la normativa establecida y que, como se ha mencionado, debe responder a la transparencia informativa y al protocolo en situaciones de crisis nuclear operativa. En el caso de la comunicación en situaciones de emergencia nuclear es una actividad regulada y protocolizada donde la responsabilidad en la comunicación la asumen organismos oficiales (Cobos \& Recoder (2019a).

Los responsables de comunicación de las centrales nucleares españolas apuntan, en las entrevistas realizadas, que las plantas atómicas -desde su construcción- han buscado, a través de la comunicación, obtener la confianza del público en general y de los diferentes grupos de interés que tienen capacidad de decidir sobre el futuro de la actividad comercial.

Para buscar esa aceptación pública de la energía nuclear, en primer lugar parece necesario realizar una actividad comercial responsable, y luego contárselo a los diferentes grupos de interés a través de la comunicación y el diálogo público (Cobos, 2017), teniendo presente que algunos stakeholders, como por ejemplo los medios de comunicación, tienen influencia en la percepción pública de la energía nuclear (Cobos \& Recoder, 2019c). Godall (2016) entiende esta comunicación como una "responsabilidad" que deben asumir las plantas atómicas con los distintos públicos.

Y para que esta orientación estratégica de la comunicación -basada en el diálogo-surta efecto, las centrales nucleares deben emplear de manera adecuada las diferentes herramientas y canales informativos para lograr alcance, como pudieran ser el caso de las redes sociales (Cobos, 2021) y la página web (Cobos \& Recoder, 2019b).

Ese derecho a la información que tienen los ciudadanos y esa necesidad que tiene la industria nuclear de ser aceptada por los stakeholders críticos nos hace reflexionar sobre la importancia de la transparencia informativa que rodea a los agentes responsables de la Comunicación Nuclear. Como señala Ruiz de Apodaca (2011:71): "Es absolutamente necesaria, la información y la transparencia por parte de los gobiernos, de los organismos reguladores y de las propias empresas sobre las ventajas e inconvenientes de la energía nuclear".

Por otra parte, algunas entidades internacionales con prestigio en el sector atómico como el Organismo Internacional de la Energía Atómica (IAEA), el Instituto Nuclear de la Energía (NEI) o la Asociación Mundial de Operadores Nucleares (WANO) sí que disponen de algunas recomendaciones de buenas prácticas en asuntos de Comunicación Nuclear, aunque en ningún caso son normativas.

La IAEA dispone de un manual de uso para que las centrales nucleares puedan comunicarse con el público en caso de emergencia nuclear o radiológica. Es un documento con recomendaciones para orientar la comunicación en caso de accidentes, pero en ningún caso es impositivo. En este manual se recogen asuntos como la organización de la comunicación en la emergencia, funciones de cada organismo, una guía de actuación para las comunicaciones públicas o reseñas informativas. Este Organismo también dispone de un manual de Comunicación Nuclear con recomendaciones prácticas para situaciones regulares aunque no se ha renovado desde 1994. 
Por su parte, el NEI también dispone de una guía de recomendaciones prácticas -no normativas- para la difusión de la información al público por parte de la industria nuclear en caso de accidentes.

\section{Conclusiones}

La comunicación en el sector atómico es necesaria según indican los responsables de comunicación de las centrales nucleares de España (Cornadó, 2016; Alcázar, 2016; Godall, 2016; Gómez, 2017; Sala, 2017): "Esa actividad industrial tiene que estar justificada e informada y ahí aparece la comunicación como herramienta imprescindible" (Cruz, 2017).

Los protagonistas de la Comunicación Nuclear en España entienden que la actividad comunicativa ha de ser estratégico-táctica debido a la "connotación social y política" (Godall, 2016) que rodea al sector atómico. Y ese diálogo multistakeholder debe ir ligado a la divulgación de la Ciencia Nuclear, con el objetivo de reparar esa desinformación que tiene la población y que reflejan las encuestas de opinión (Cobos, 2017; Alcázar, 2016).

La revisión de toda la legislación nuclear así como el conocimiento de la praxis comunicativa de las plantas atómicas en España-gracias al testimonio de los responsables de comunicación-nos permite afirmar que las plantas nucleares tienen libertad para organizar la comunicación con sus grupos de interés en situaciones de actividad ordinaria, ya que la ley les exige, de manera general, transparencia y acceso a la información con los ciudadanos.

Sin embargo, las plantas atómicas tienen que informar al organismo regulador sobre cualquier acontecimiento que se produzca en las instalaciones nucleares (Cobos \& Recoder, 2019a). La comunicación de crisis sí que está reglada en caso de incidentes o accidentes, no así en otras situaciones que no entrañan riesgo para la salud de las personas o el medio ambiente pero que tienen que ver con sucesos críticos para la organización (por ejemplo, eventos que pueden dañar la imagen de la empresa o el sector pero que no pertenecen a sucesos operativos, como pudiera ser el caso de manifestaciones de grupos ecologistas o antinucleares, o la repercusión en prensa de una declaración en contra de esta fuente de energía, entre otros) donde la responsabilidad de la comunicación recae sobre cada central nuclear o la empresa que la respalda.

Por lo tanto y respondiendo a la primera pregunta de investigación, las centrales nucleares de España y las empresas responsables de su operación, tienen autonomía real para organizar la comunicación en situaciones normales aunque deben atender a la transparencia informativa que marca la legislación. Además, las plantas atómicas pueden dirigir la comunicación de crisis de sucesos no operativos. Sin embargo, en el caso de crisis nucleares las riendas de la comunicación las asumen las autoridades competentes en España siguiendo el protocolo que está preestablecido.

Por otra parte, y respondiendo a la segunda pregunta de investigación, la legislación cubre al ciudadano a nivel informativo exclusivamente en las situaciones de crisis nuclear operativa, ya que se recogen los diferentes escenarios que pueden ocurrir, la información que debe ser suministrada a la opinión pública, y quien es la autoridad responsable de informar. Sin embargo, en aquellas situaciones alejadas de crisis nucleares, no existe una protección de la legislación nuclear a nivel informativo por la ausencia de un desarrollo más amplio y concreto de la normativa. En este sentido, la ley plantea de manera general la transparencia como principio de la comunicación con el público y no se recogen propuestas comuni- 
cativas más concretas y un control de las mismas en las diferentes situaciones que pueden suceder en una central nuclear en períodos de actividad ordinaria.

Para velar por los intereses de la población y en favor de la transparencia, creemos conveniente que todos los agentes que intervienen en el engranaje nuclear deben establecer un compromiso auténtico con la sociedad a través de la disposición informativa. Se trata de relegar a un segundo plano los intereses económicos, políticos e ideológicos para hacer más comprensible esta industria, y señalar los beneficios y los riesgos que conlleva la actividad atómica, para que las decisiones que se tomen se realicen por el bien común de todos.

Pero para poder materializar este punto, entendemos que el primer paso es establecer a nivel normativo una base más desarrollada e impositiva asegurando al ciudadano el acceso a una información transparente. Creemos que si no sucede así los ciudadanos estarán desprotegidos ante industrias cuya producción implique riesgos, como es el caso de las centrales nucleares.

Y consideramos que si existe un mayor desarrollo de la legislación en Comunicación Nuclear, la confianza de la sociedad en las instituciones podría ser mayor, y mayor podría ser la credibilidad que cada ciudadano otorgue a la autoridad informativa. Este punto puede contribuir a la aceptación pública de la energía nuclear.

Por todo lo indicado, la transparencia informativa es un requisito básico de derecho, una responsabilidad que no se puede eludir, pero sobre todo un compromiso pleno con la ciudadanía.

\section{Referencias bibliográficas}

Abe, Y. (2013). Risk Assessment of Nuclear Power by Japanese Newspapers Following the Chernobyl Nuclear Disaster. International Journal of Communication, 7, 1968-1989.

Alcázar, J. P. Jefe de Comunicación de CNAT. Entrevista en profundidad. Sede corporativa Centrales Nucleares Almaraz-Trillo (Madrid), 21 de diciembre de 2016.

Centro de Investigaciones Sociológicas. Barómetros (mayo 2018, septiembre 2017, julio 2017, noviembre 2015, diciembre de 2015, mayo 2011, mayo 2010, marzo 2007, noviembre 2007, septiembre 2006, diciembre 2004, diciembre 2003, diciembre 2002, diciembre 2001, abril 2000, diciembre 2000, mayo 1997, noviembre 1997, febrero 1996, febrero 1995, diciembre1993, diciembre 1992, noviembre 1990, diciembre 1991, julio 1991, junio 1978). Disponible en: www.cis.es.

Cobos, E. (2021). Social Media: useful for high-risk industries? A study of nuclear energy in Spain. Artículo aceptado en Communication \& Society, Universidad de Navarra.

Cobos, E. yRecoder, M.J. (2019a).La comunicación de crisisen el sector atómico:protocolo, características, yrecomendaciones en eventos nucleares. Revista Española de Comunicación en Salud (RECS) -Monográfico sobre Periodismo y comunicación en situaciones de crisis, emergencias y desastres-, 19-29. DOI: https://doi.org/10.20318/recs.2019.4372

Cobos, E. y Recoder, M. J. (2019b). Modelo de análisis web para centrales nucleares: estudio del caso de España. Estudios Sobre el Mensaje Periodístico, 25 (2), 727-745. DOI: https://doi.org/10.5209/esmp.64799 
Cobos, E. y Recoder, M.J. (2019c). La imagen de la energía nuclear en la prensa española de proximidad: ¿Son los medios de comunicación un stakeholder relevante para el sector atómico? Anàlisi: Quaderns de comunicació i cultura, 61, 31-53. DOI: https://doi.org/10.5565/rev/analisi.3181

Cobos, E. (2017). La energía nuclear en España y el bien común: el diálogo como propuesta comunicativa para la aceptación social. Ruta Comunicación, 8, 27-44.

Comisión Europea. Barómetros (septiembre 2017, agosto 2017, abril 2015, mayo 2015, enero 2013, octubre 2011, marzo 2010, junio 2008; febrero 2007; enero 2006; septiembre 2005; diciembre 2002; abril 2002). Disponible en: https://bit.ly/3oyoely.

Convenio de Aarhus. Disponible en: https://bit.ly/3mmSiyo.

Cornadó, A. Director de Comunicación de Nuclenor y Presidente del Foro Nuclear (2013-2017). Entrevista en profundidad. Foro de la Industria Nuclear Española (Madrid), 21 de diciembre de 2016.

Cornadó, A. (2006). La comunicación pública y la energía nuclear. Nuclear España, 261, pp. 32-36.

Cruz, J. Responsable de Comunicación de Cofrentes (1984-2015). Entrevista en profundidad. Valencia, 16 de febrero de 2017. Declaración de Río de Janeiro (1992). Disponible en: https://bit.ly/31N569M.

Declaración de Río de Janeiro (2012). Disponible en: https://bit.ly/3dZzPFn.

De Oliveira, C. (2015). Comunicación y percepción del riesgo. El periodismo en España en el aniversario del accidente nuclear de Fukushima Daiichi. Tesis. Pamplona: Universidad de Navarra.

Directiva 2014-87-EURATOM, del Consejo, de 8 de julio de 2014, por la que se modifica la Directiva 2009-71-EURATOM del Consejo, de 25 de junio de 2009, por la que se establece un marco comunitario para la seguridad nuclear de las instalaciones nucleares. Disponible en: https://bit.ly/3msnqwF.

Directiva 2011/70/EURATOM, del Consejo, de 19 de julio de 2011, por la que se establece un marco comunitario para la gestión responsable y segura del combustible nuclear gastado y de los residuos radiactivos. Disponible en: https://bit. ly/37LnJP9.

Godall, M. Directora de Comunicación de ANAV. Entrevista en profundidad. Central Nuclear de Vandellós II (Tarragona), 3 de noviembre de 2016.

Gómez, C. Responsable del Centro de Información de Cofrentes. Entrevista en profundidad. Central Nuclear de Cofrentes, 17 de febrero de 2017.

IAEA (2012). Manual de Operaciones para la Comunicación de Incidentes y Emergencias. Vienna: International Atomic Energy Agency. Disponible en: https://bit.ly/3oHbEAy.

IAEA (1994). Nuclear communications: a handbook for guiding good communications practices at nuclear fuel cycle facilities. Vienna: International Atomic Energy Agency. Disponible en: https://bit.ly/3mraK9d.

Ipsos Consulting e Innova Investigación de Mercados. Encuesta de opinión pública ante la energía nuclear en España (2014-2018). 
Instrucción IS-10, revisión 1, de 30 de julio de 2014, del Consejo de Seguridad Nuclear, por la que se establecen los criterios de notificación de sucesos al Consejo por parte de las centrales nucleares. Disponible en: https://bit.ly/2TwvfVH.

Instrucción IS-19, de 22 de octubre de 2008, del Consejo de Seguridad Nuclear, sobre los requisitos del sistema de gestión de las instalaciones nucleares. Disponible en: https://bit.ly/2HJmVlW.

Ionescu, T. B. (2012). Communicating in Germany about the Fukushima Accident; How Direct Encounter Beat Media Representations. Environmental Communication, 6 (2), 260-267. DOI: https://doi.org/10.1080/17524032.2012.672443

Koerner, C. L. (2014). Media, fear, and nuclear energy: A case study. The Social Science Journal, 51, 240-249. DOI: http:// dx.doi.org/10.1016/j.soscij.2013.07.011

Ley 27/2006, de 18 de julio, por la que se regulan los derechos de acceso a la información, de participación pública y de acceso a la justicia en materia de medio ambiente. Disponible en: https://bit.ly/3e35eGW.

Ley 25-1964, de 29 de abril, sobre energía nuclear. Disponible en: https://bit.ly/3e3Ai9j.

Ley 12-2011, de 27 de mayo, sobre responsabilidad civil por daños nucleares o producidos por materiales radiactivos. Disponible en: https://bit.ly/3ovZFp7.

Ley 15-1980, de 22 de abril, de creación del Consejo de Seguridad Nuclear. Disponible en: https://bit.ly/31KGcqY.

Ley 33/2007, de 7 de noviembre de 2007 que modifica la Ley 15-1980 de creación del CSN. Disponible en: https://bit. ly/3e0x46F.

Ley 19-2013, de 9 de diciembre, de transparencia, acceso a la información y buen gobierno. Disponible en: https://bit. ly/31KqRXK.

Lu, Y. (2012). Comparative analysis of nuclear crisis communication: 2011 Fukushima nuclear crisis and 1979 Three Mile Island nuclear crisis. Tesis. California: University of Southern California.

Melo, A. Director de Organización y Recursos Humanos de CNAT. Entrevista por mail, 25 de enero de 2017.

Muñoz, N. (2012). La seguridad nuclear en el siglo XXI: transparencia y pedagogía informativas. Economía Industrial, 384, 33-36.

NEI (2014). Implementing and Operating a Joint Information System. Washington D.C.: Nuclear Energy Institute. Disponible en: https://bit.ly/3mrblrt.

PENBU - Plan de Emergencia Nuclear Exterior a la Central Nuclear de Santa María de Garoña (Burgos). Disponible en: https://bit.ly/35I1F56.

PENCA - Plan de Emergencia Nuclear Exterior a la Central Nuclear de Almaraz (Cáceres). Disponible en: https://bit. ly/2G46InR.

PENGUA - Plan de Emergencia Nuclear Exterior a las Centrales Nucleares de José Cabrera y Trillo (Guadalajara). Disponible en: https://bit.ly/3jsyyrq. 
PENTA - Plan de Emergencia Nuclear Exterior a las Centrales Nucleares de Ascó y Vandellós (Tarragona). Disponible en: https://bit.ly/3mrJebD.

PENVA - Plan de Emergencia Nuclear Exterior a la Central Nuclear de Cofrentes (Valencia). Disponible en: https://bit. ly/3oyulGk.

Perko, T. (2011). Importance of risk communication during and after a nuclear accident. Integrated Environmental Assessment \& Management, 7(3), 388-392. DOI: https://doi.org/10.1002/ieam.230

Perko, T., Turcanu, C. y Carlé, B. (2012). Media Reporting of Nuclear Emergencies: The Effects of Transparent Communication in a Minor Nuclear Event. Journal of Contingencies \& Crisis Management, 20 (1), 52-63. DOI: http://dx.doi. org/10.1111/j.1468-5973.2012.00663.x

Perko, T., Gorp, B., Turcanu, C., Thijssen, P. y Carle, B. (2013). Communication in Nuclear Emergency Preparedness: A Closer Look at Information Reception. Risk Analysis: An International Journal, 33 (11), 1987-2001. DOI: https://doi.org/10.1111/ risa. 12048

Real Decreto 393-2007, de 23 de marzo, por el que se aprueba la Norma Básica de Autoprotección de los centros, establecimientos y dependencias dedicados a actividades que puedan dar origen a situaciones de emergencia. Disponible en: https://bit.ly/31MWnEa.

Real Decreto 243-2009, de 27 de febrero, por el que se regula la vigilancia y control de traslados de residuos radioactivos y combustible nuclear gastado entre Estados miembros o procedentes o con destino al exterior de la Comunidad. Disponible en: https://bit.ly/35D4btB.

Real Decreto 1546/2004, de 25 de junio, por el que se aprueba el Plan Básico de Emergencia Nuclear. Disponible en: https:/ / bit.ly/37Mw1X2.

Ruiz de Apodaca, Á. M. (2011). Nuevas perspectivas del Derecho nuclear en Europa y en España. REDUR, 9, 67-98. DOI: https://doi.org/10.18172/redur.4082

Ruiz de Apodaca, Á. M. (2010). Régimen de la energía nuclear a la luz del Derecho Comunitario: competencia, seguridad, aceptación y perspectivas. Noticias de la Unión Europea, 322, 47-58.

Sala, J. Portavoz de Cofrentes y Jefe de Producción. Entrevista en profundidad. Central Nuclear de Cofrentes, 17 de febrero de 2017 .

Siegrist, M. y Visschers, V.H.M. (2013). Acceptance of nuclear power: The Fukushima effect. Energy Policy, 59, 112-119. DOI: https://doi.org/10.1016/j.enpol.2012.07.051

Yamamura, E. (2012). Effect of Free Media on Views Regarding Nuclear Energy after the Fukushima Accident. Kyklos, 65 (1), 132-141. DOI: http://dx.doi.org/10.1111/j.1467-6435.2011.00530.x 